\title{
RAMAN SCATTERING IN $\mathrm{Zn}_{1-x} \mathrm{Mg}_{x}$ Se MIXED CRYSTALS*
}

\section{Kozielski, M. SzYbowicz}

Institute of Physics, Poznań Technical University, Piotrowo 3, 60-965 Poznań, Poland

F. FIRSZT, S. ŁĘGowsKi, H. MĘCZYŃSKa, B. SEKULSKA, J. SZA'TKoWSKI ${ }^{\dagger}$

Institute of Physics, N. Copernicus University, Grudziądzka 5/7, 87-100 Torun, Poland

AND W. PASzKowICZ

Institute of Physics, Polish Academy of Sciences

Al. Lotników 32/46, 02-668 Warsaw, Poland

This paper presents results of investigations of the Raman scattering of vibrational modes in $\mathrm{Zn}_{1-x} \mathrm{Mg}_{x}$ Se crystals for the range of composition $0<x<0.43$. The Raman polarized spectra of $\mathrm{Zn}_{1-x} \mathrm{Mg}_{x} \mathrm{Se}$ were measured at room temperature. The typical Raman spectrum shows four peaks which can be interpreted as longitudinal and transverse optical $\mathrm{ZnSe-like}$ and MgSe-like phonons. The $\mathrm{ZnSe}$-like and MgSe-like vibrations can be described by the modified random element isodisplacement model. Results of the Raman measurements in the investigated range of composition confirm the two-mode behaviour of the optical phonons in the mixed $\mathrm{Zn}_{1-x} \mathrm{Mg}_{x} \mathrm{Se}$ crystals.

PACS numbers: 78.30.Fs, 78.55.Et, 63.20.-e

The physical properties of wide gap II-VI ternary and quaternary mixed crystals have been recently extensively studied. The current interest in these materials arises from scientific and application points of view. As far as an application is concerned, ternary and quaternary II-VI alloys are especially interesting in the context of possibility of an additional control of band gap energies, lattice constants as well as conduction and valence bands offsets in heterostructures. These parameters are very important for effective carriers injection and radiative recombination processes in electroluminescent devices based on multilayer heterostructures.

For the study of vibrational properties of mixed crystals the Raman scattering is widely used as the experimental method. The vibrational spectra of tetrahedrally coordinated semiconductors where a fraction of cations is replaced by an

* This work is supported in part by the grant no. DS 62-120/4 of Poznan Technical University and by grant no. 326-F of $N$. Copernicus University.

$t_{A}$ A fellowship given to J.S. by the Foundation for Polish Science is gratefully acknowledged. 
element, which has an atomic mass significantly smaller than this of constituent atoms of a host lattice, can be described by two-mode model $[1,2]$. This lighter component exhibits a vibrational mode (local mode) with a frequency higher than that of the perfect crystal. Such behaviour can be expected for $\mathrm{Zn}_{1-x} \mathrm{Mg}_{x}$ Se mixed crystal which is lately extensively studied because of potential application in constructing blue and UV light sources [3-6]. However, no experimental data for $\mathrm{Zn}_{1-x} \mathrm{Mg}_{x}$ Se bulk crystals are available until now. Some data for epilayers grown on GaAs have been recently published [7].

This paper deals with experimental study of compositional dependence of the Raman spectra in $\mathrm{Zn}_{1-x} \mathrm{Mg}_{x}$ Se solid solution. Mixed $\mathrm{Zn}_{1-x} \mathrm{Mg}_{x}$ Se crystals were grown by modified Bridgman method described in [4]. The composition was determined by means of electron probe microanalysis. It has been already established that $\mathrm{Zn}_{1-x} \mathrm{Mg}_{x}$ Se crystallises in sphalerite form for $x<0.185$, but for higher $x$ value in wurtzite one. This sphalerite-wurtzite transition seems to be typical of $(\mathrm{AM})^{\mathrm{II}} \mathrm{B}^{\mathrm{VI}}$ systems where $\mathrm{M}$ is a metal with a vanishing or low number of $3 d$ electrons like $\mathrm{Mg}$. It has been also observed in $\mathrm{Zn}_{1-x} \mathrm{Mg}_{x} \mathrm{Te}[8,9], \mathrm{Cd}_{1-x} \mathrm{Mg}_{x} \mathrm{Te}$ $[10]$ and $\mathrm{Zn}_{1-x} \mathrm{Mg}_{x} \mathrm{Se}[11,12]$. Some preliminary results on photoluminescence and electrical properties of $\mathrm{Zn}_{1-x} \mathrm{Mg}_{x} \mathrm{Se}$ bulk crystals have been already published $[5,6]$.

The Raman polarized spectra were measured at room temperature in quasi-backscattering geometry [13]. As a source of radiation an argon ion laser tuned at $488 \mathrm{~nm}$ was used. The scattered light was observed at right angle to the incident beam and its spectrum was analysed with Zeiss GDM-1000 double grating monochromator. The radiation was detected by cooled EMI 9658B photomultiplier followed by a photon counter. The entire measuring system was controlled by computer which was responsible for experimental data acquisition and further numerical analysis. The program of the non-linear regression was applied for optimising the fit of Lorentzian functions to the observed Raman band profiles. The experimental setup permitted to estimate the band positions in the Raman spectra with an accuracy $\pm 2 \mathrm{~cm}^{-1}$.

The room temperature vibrational Raman spectra for $\mathrm{Zn}_{1-x} \mathrm{Mg}_{x}$ Se with two different $\mathrm{Mg}$ concentrations are shown in Fig. 1. Numerical fit of the experimental Raman band shapes to Lorentzian functions is also presented. The typical spectrum consists of $\mathrm{ZnSe}$-like and MgSe-like transverse (TO) and longitudinal (LO) phonon peaks. In some samples with low $\mathrm{Mg}$ content $\mathrm{MgSe}$-like peaks are weakly resolved.

The half-width of the Raman phonon peaks increases with the increase in $\mathrm{Mg}$ content (Fig. 2). The peak positions and line widths of the Raman spectra are determined by alloy disorder due to statistical distribution of local cation concentrations $[14,15]$. Alloy potential fluctuations remove the translational invariance of lattice, resulting in the breakdown of Raman selection rule and causing a broadening of the Raman peaks.

The behaviour of transverse and longitudinal modes in $\mathrm{Zn}_{1-x} \mathrm{Mg}_{x} \mathrm{Se}$ mixed crystals as a function of $x$ is quite well described by the modified random element isodisplacement (MREI) model proposed for $\mathrm{AB}_{1-x} \mathrm{C}_{x}$ compounds by Chang and Mitra [1]. 


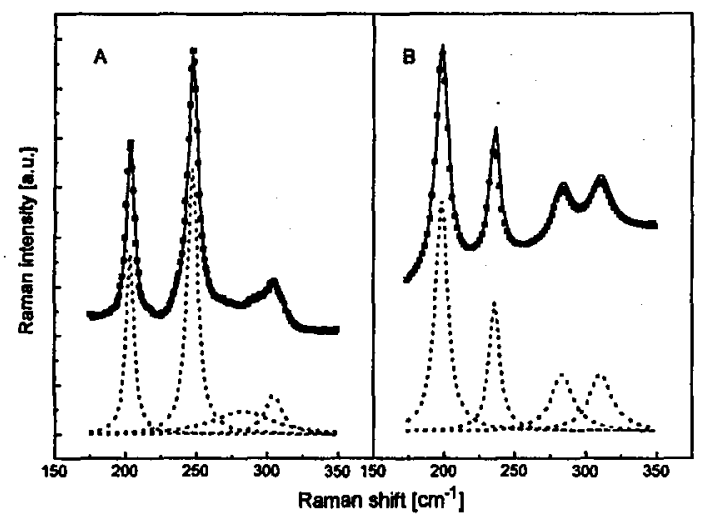

Fig. 1. Room temperature Raman spectra of $\mathrm{Zn}_{1-x} \mathrm{Mg}_{x}$ Se crystals with two different magnesium content: (A) $x=0.06$, (B) $x=0.34$, squares - experimental points, solid lines - the fit to Lorentzian components indicated with dotted lines.

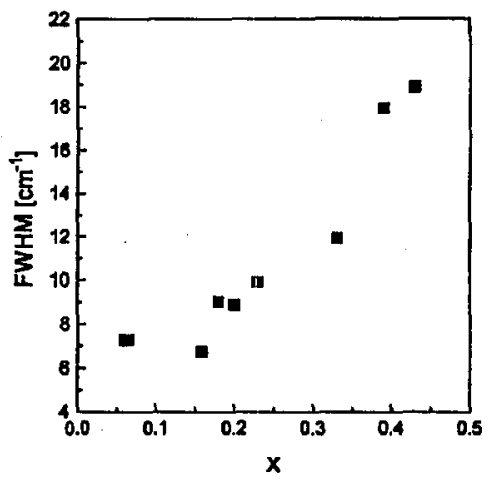

Fig. 2. The full width at half maximum of $\mathrm{TO}(\mathrm{ZnSe})$ mode in $\mathrm{Zn}_{1-x} \mathrm{Mg}_{x} \mathrm{Se}$ as a function of composition.

The $\mathrm{Zn}_{1-x} \mathrm{Mg}_{x}$ Se semiconductor shows a typical two-mode behaviour. The necessary condition for such behaviour is the existence of local and gap modes in the limit of small concentration of the light and heavy constituents, where each TO-LO mode pair for the binary compounds degenerates to an impurity mode (local mode for $\mathrm{Mg}$ in $\mathrm{ZnSe}$ and gap mode for $\mathrm{Zn}$ in $\mathrm{MgSe}$ ). This is in agreement with the mass criterion according to which for relation $m_{\mathrm{C}}<m_{\mathrm{B}}, m_{\mathrm{C}}<m_{\mathrm{A}}$ and $m_{\mathrm{C}}<\mu_{\mathrm{BC}}$, where $\mu_{\mathrm{BC}}$ is the reduced mass $\left(\mu_{\mathrm{BC}}=\left(1 / m_{\mathrm{B}}+1 / m_{\mathrm{C}}\right)^{-1}\right)$, two-mode behaviour should be observed [1]. These conditions are fulfilled in the case of $\mathrm{Zn}_{1-x} \mathrm{Mg}_{x}$ Se.

Figure 3 presents positions of $\mathrm{ZnSe}-$ like and $\mathrm{MgSe}-\mathrm{like} \mathrm{LO}$ and TO modes as a function of magnesium content. The solid lines were calculated according to MREI model using literature data for pure ZnSe. Data for MgSe were estimated by fitting procedure. The measurements were performed for mixed crystals with 


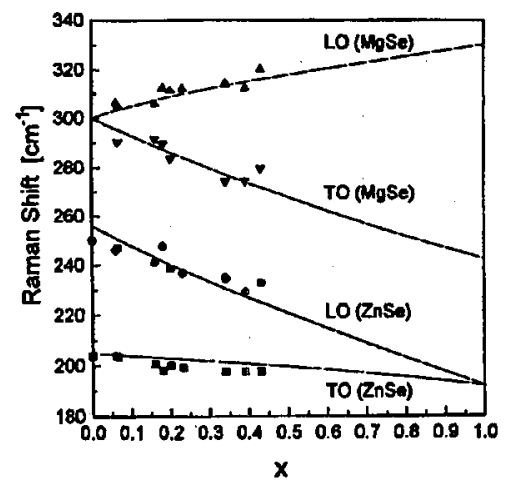

Fig. 3. The frequencies of optical phonons in $\mathrm{Zn}_{1-x} \mathrm{Mg}_{x}$ Se crystals at room temperature; up triangles - LO ( $\mathrm{MgSe})$, down triangles - TO $(\mathrm{MgSe})$, circles - LO $(\mathrm{ZnSe})$, squares - TO ( $\mathrm{ZnSe})$, solid line - the fit to MREI model (for details see text).

$x$ up to 0.6. It was not possible to obtain a crystalline $\mathrm{Zn}_{1-x} \mathrm{Mg}_{x}$ Se material for $x \geq 0.6$ by our modified Bridgman method. It should be pointed out however that for $\mathrm{Mg}$ content higher than about $50 \% \mathrm{Zn}_{1-x} \mathrm{Mg}_{x}$ Se crystals are not stable in air.

In our calculations the difference between zinc-blende and wurtzite structure of $\mathrm{Zn}_{1-x} \mathrm{Mg}_{x} \mathrm{Se}$ was neglected. The following data were taken for calculations: TO $(\mathrm{ZnSe})=205 \mathrm{~cm}^{-1}, \mathrm{LO}(\mathrm{ZnSe})=251 \mathrm{~cm}^{-1}, \varepsilon_{\infty}(\mathrm{ZnSe})=9.2$. The following linear dependence of cubic lattice constant on $\mathrm{Mg}$ content $x$ were used: $a(x)=5.67+$ $0.22 x \AA[5,6]$. In the applied MREI model we estimated all parameters of pure MgSe to fit the computed values to experimental data, since these data for MgSe are not known. From the curve fitting we estimated the gap mode frequency of $\mathrm{Zn}$ in $\mathrm{MgSe}$, static dielectric constant $\varepsilon_{0}$ as well as TO and LO phonon frequencies for pure $\mathrm{MgSe}$. It was also possible to determine the corresponding force constants $F_{\mathrm{Zn}-\mathrm{Se}}, F_{\mathrm{Zn}-\mathrm{Mg}}$ and $F_{\mathrm{Mg}-\mathrm{Se}}$. The estimated best fit parameters are as follows: TO $(\mathrm{MgSe})=237 \mathrm{~cm}^{-1}$, LO $(\mathrm{MgSe})=340 \mathrm{~cm}^{-1}, \varepsilon_{\infty}(\mathrm{MgSe})=3.8$, impurity mode $\mathrm{Mg}$ in $\mathrm{ZnSe}-300 \mathrm{~cm}^{-1}$ and impurity mode $\mathrm{Zn}$ in $\mathrm{MgSe}-192 \mathrm{~cm}^{-1}$. These physical constants for $\mathrm{MgSe}$ are in reasonable agreement with corresponding data calculated for thin films [7]. The following force constants were calculated [in units $10^{6}$ a.m.u. $\left(\mathrm{cm}^{-1}\right)^{2}$ ]: $F_{\mathrm{Zn}-\mathrm{Se}}=1.82, F_{\mathrm{Mg}-\mathrm{Se}}=1.57, F_{\mathrm{Zn}-\mathrm{Mg}}=0.3$. These values are similar to analogous parameters obtained for some wide-gap II-VI compounds, e.g. $(\mathrm{Zn}, \mathrm{Mn}) \mathrm{Se},(\mathrm{Cd}, \mathrm{Mn}) \mathrm{Se}$ [12], $(\mathrm{Zn}, \mathrm{Mn}) \mathrm{Te}$ and $(\mathrm{Cd}, \mathrm{Mn}) \mathrm{Te}$ [2].

As the fraction of $\mathrm{Mg}$ increases, experimental data of the Raman shift for MgSe-like and ZnSe-like modes deviate from the calculated ones. This can be explained if one takes into account that the hexagonal crystal field in wurtzite structure splits vibrational modes of zinc-blende lattice [11]. From fitted curves (Fig. 2) one can conclude that a larger LO-TO splitting is for MgSe than for $\mathrm{ZnSe}$. This is probably caused by more ionic character of chemical bonds in $\mathrm{MgSe}$ than in $\mathrm{ZnSe}$, which influences the screening of the electronic Coulomb interactions $[7,16]$. 
From presented in Fig. 3 dependence of energetical positions of ZnSe-like and MgSe-like LO and TO modes on magnesium content one can see that measurements of the Raman spectra can be used as a non-destructive method for estimating of composition of $\mathrm{Zn}_{1-x} \mathrm{Mg}_{x}$ Se crystals.

\section{References}

[1] I.F. Chang, S.S. Mitra, Adv. Phys. 20, 359 (1971).

[2] D.L. Peterson, A. Petrou, W. Giriat, A.K. Ramdas, S. Rodriguez, Phys. Rev. B 33, 1160 (1986).

[3] S.O. Ferreira, H. Sitter, W. Faschinger, R. Krump, G. Brunthaler, J. Cryst. Growth 146, 418 (1995).

[4] J. Szatkowski, F. Firszt, H. Męczyńska, S. Łęgowski, Acta Phys. Pol. A 84, 531 (1993).

[5] F. Firszt, H. Męczyńska, B. Sekulska, J. Szatkowski, W. Paszkowicz, J. Kachniarz, Semicond. Sci. Technol. 10, 197 (1995).

[6] F. Firszt, S. Łęgowski, H. Męczyńska, H.L. Oczkowski, W. Osińska, J. Szatkowski, W. Paszkowicz, Z.M. Spolnik, J. Cryst. Growth 159, 167 (1996).

[7] D. Huang, C. Jin, D. Wang, X. Liv, Appl. Phys. Lett. 67, 3611 (1995).

[8] G. Revel, J.L. Pastol, J.C. Rouchaud, M. Fédoroff, J.C. Guillaume, J. Chevallier, J.F. Rommeluere, J. Electron. Mater. 9, 165 (1980).

[9] S.G. Parker, A.R. Reinberg, J.E. Pinnel, W.C. Holton, J. Electrochem. Soc. 118, 979 (1971).

[10] K. Itoh, in: II-VI Semiconducting Compounds, Ed. D.G. Thomas, Benjamin, New York 1967, p. 1296.

[11] M. Jain, J.L. Robbins, in: Diluted Magnetic Semiconductors, Ed. M. Jain, World Scientific, Singapore 1991, p. 1.

[12] P.D. Lao, Y. Guo, G. Siu, S.C. Chen, Phys. Rev. B 48, 11701 (1993).

[13] B. Susła, M. Drozdowski, M. Kozielski, St. Szuba, Acta Phys. Pol. A 73, 369 (1988).

[14] P. Paraynthal, F. Pollak, Phys. Rev. Lett. 52, 1822 (1983).

[15] W. Meredith, G. Horsburgh, G.D. Brownlie, K.A. Prior, B.C. Cavanett, W. Rothwell, A.J. Dann, J. Cryst. Growth 159, 103 (1996).

[16] N.W. Ashcroft, N.D. Mermin, Solid State Physics, Saungers College, Philadelphia 1976. 